\title{
Electrocardiographic Changes in Hypertensive Crisis in a Tertiary Care Hospital
}

\author{
Pavan D. Patel ${ }^{1}$, Shruti Nair², D. M. Patel ${ }^{3}$, Nitin Jadhav ${ }^{4}$ \\ ${ }^{1}$ Resident, Department of Medicine, KIMS, Karad, Maharashtra, India. ${ }^{2}$ Resident, Department of Obstetrics and \\ Gynaecology, KIMS, Karad, Maharashtra, India. ${ }^{3}$ Associate Professor, Department of Medicine, Vedanta Institute of \\ Medical Sciences, Palghar, Maharashtra, India. ${ }^{4}$ Associate Professor, Department of Medicine, KIMS, Karad, \\ Maharashtra, India.
}

\section{ABSTRACT}

\section{BACKGROUND}

Cardiovascular disorders are the leading causes of disability and death worldwide, and a great majority of Cardiovascular Disorders are associated with dyslipidemia. Worldwide, there is broad variation in serum lipid profile levels among different population groups. Increased serum levels of total cholesterol, triglycerides, low density lipoproteins and decreased high density lipoproteins are known to be major risk factors for cardiovascular disorders. The present study was conducted to assess the lipid levels among cases of accelerated hypertension presenting to a tertiary healthcare institute.

\section{METHODS}

The present study was a cross sectional observational study done on 96 patients who presented with hypertensive emergencies. They were admitted under Department of Medicine, KIMS, Karad, from August 2018 to December 2018. On admission, detailed history was taken, and complete clinical examination was done. It was a hospital based cross sectional study.

\section{RESULTS}

Serum levels of TC, TG, HDL and LDL in hypertensives were $182 \pm 3.4,143.62 \pm 6.3$, 49.68 and $95.83 \pm 7.8 \mathrm{mg} / \mathrm{dL}$, respectively.

\section{CONCLUSIONS}

Our results suggest that elevated BP may predict certain disturbances in lipoprotein metabolism.

\section{KEYWORDS}

Accelerated Hypertension, Hypertensive Crisis, Lipid Profile, Blood Pressure, Total Cholesterol, Triglycerides, HDL, LDL
Corresponding Author:

Dr. Pavan D. Patel,

Department of Medicine,

Krishna Institute of Medical Sciences,

Karad, Maharashtra, India.

E-mail: pavan_patel10574@icloud.com

DOI: 10.14260/jemds/2019/586

Financial or Other Competing Interests: None.

How to Cite This Article:

Patel PD, Nair S, Patel DM, et al. Electrocardiographic changes in hypertensive crisis in tertiary care hospital. Evolution Med. Dent. Sci. 2019;8(35): 2698-2702,DOI:

10.14260/jemds/2019/586

Submission 21-03-2019,

Peer Review 15-08-2019,

Acceptance 22-08-2019,

Published 02-09-2019.

\section{(i) (3)}




\section{BACKGROUND}

Raised blood pressure and deranged lipid levels are the major risk factors for development of non communicable diseases like cardiovascular disease. Hypertensive disorders are responsible for nearly mortality of $80 \%$ and loss of DALY among SEAR countries, the prevalence is reported to be increased in developing countries. ${ }^{1,2}$ In developing countries, with communicable disease, non-communicable diseases causes double burden of diseases. Previous researches have described the relationship between development of hypertension and deranged lipid levels. Its pathophysiology have already been studied.

\section{Epidemiology}

The first large study 3 of the natural history of hypertensive emergencies was published in 1939. The results of this seminal article revealed that untreated hypertensive emergencies had a 1-year mortality rate of $79 \%$, with a median survival of 10.5 months. Before the introduction of antihypertensive medications, approximately $7 \%$ of hypertensive patients had a hypertensive emergency 4 . Currently, with the improvement in the drugs available for the treatment of hypertension, it is estimated that 1 to $2 \%$ of patients with hypertension will have a hypertensive emergency at some time in their life.5,6 Despite the development of increasingly effective antihypertensive treatments over the past 4 decades, the incidence of hypertensive emergencies has increased. ${ }^{7}$ The vast majority of patients who present with a hypertensive emergency to an emergency department have been previously diagnosed with hypertension and have been prescribed antihypertensive agents. ${ }^{8,9}$ However, in many of these patients BP control prior to presentation was inadequate. In some studies, ${ }^{10}$ up to $50 \%$ of patients who presented to an emergency department with hypertensive emergency were not adherent with their regimen in the preceding week. Illicit drug use has been reported $^{11}$ to be a major risk factor for the development of hypertensive emergency.

\section{Aetiology}

Ninety-five per cent of patients have essential hypertension with no underlying cause. The remaining $5 \%$ of patients have an underlying cause for their elevated blood pressures, of which certain groups have higher chances of presenting with a hypertensive crisis. Use of recreational drugs is a frequent cause of hypertensive crisis. Cocaine amphetamines, phencyclidine hydrochloride, and diet pills are sympathomimetic and thus may cause severe acute hypertension. Patients taking monoamine oxidase inhibitors along with tricyclic antidepressants, antihistamines, or food with tyramine are prone to hypertensive crises. Withdrawal syndromes from drugs such as clonidine or beta-blockers may also precipitate hypertensive crises. ${ }^{12}$ Pheochromocytoma is a rare cause of hypertensive crises. Patients with spinal cord disorders, such as Guillain Barre' syndrome, are also at a higher risk for hypertensive crises. Rapid industrialization, increased access to improved health care is responsible for increased life expectancy. However sedentary lifestyle, consumption of unhealthy diet, and increased prevalence of addiction among people is observed among the developing countries and it reflected as a risk factor for augmentation of the disease process. ${ }^{13}$ It is well known that raised low density lipoproteins, very low density lipoproteins, cholesterol, and triglycerides is associated with adverse outcomes in cardiovascular disorders, however high density lipoproteins is associated with favourable outcomes. ${ }^{14}$ Various studies have demonstrated and proved the causal relationship between lipid levels and development of hypertension and coronary artery disease. ${ }^{15}$

The Electrocardiogram is considered as one of the tools used in the diagnostic workup of hypertensive crisis to diagnose myocardial ischemia or infarction, typically T-wave inversion and in more severe cases, ST segment displacement. These changes mirror cardiac injury and indicate a hypertensive emergency situation and therefore necessitate prompt medical intervention. ${ }^{16}$ Existing Previously conducted researches have concluded that the diastolic left ventricular dysfunction may be the earliest detectable pathology that may be seen before left ventricular hypertrophy voltage criteria which is usually seen in a standard 12-lead ECG (Electrocardiogram). Ventricular activation time can be used to predict the diastolic dysfunction and left ventricular stiffness, representing a new marker for early diagnosis of cardiovascular disorders. ${ }^{7-19}$

The present study was conducted to assess the electrocardiographic changes among cases of accelerated hypertension presenting to a tertiary healthcare institute.

\section{METHODS}

The present study was a cross sectional observational study conducted among all the cases fulfilling inclusion criteria and willing to participate in the present study (Universal sampling). 96 patients presented with hypertensive emergencies admitted under department of medicine, KIMS, Karad, from August 2018 to December 2018. On admission, detailed history was taken and complete clinical examination was done. The study was conducted among subjects after taking their consents. The present study was done after getting approval from the institutional ethical committee.

\section{Inclusion Criteria}

1. Systolic blood pressure of $180 \mathrm{mmHg}$ and above or diastolic blood pressure of $120 \mathrm{mmHg}$ and above.

2. Evidence of end organ damage, either clinically or laboratory findings.

\section{Exclusion Criteria}

1. Patients less than 18 yrs. and above 40 yrs. of age

2. Patients with valvular heart disease, pregnancy induced hypertension.

\section{Diagnostic Criteria}

The diagnosis of hypertensive emergency will be established by the following 1 . Systolic blood pressure $\geq 180 \mathrm{mmHg}$ or diastolic blood pressure $\geq 120 \mathrm{mmHg}$. 2. Acute target organ damage. We obtained written informed consent in all cases to participate in the study.

\section{Data Collection Procedure}

All the study subjects who fulfilled the inclusion criteria were included in the present study. The consent was taken from 
the patients or attendants who were included in the study, for performing the necessary investigations or procedures. A semi-structured, pre-validated, standard case record proforma was used in the current study to collect the data. Detailed history was taken, clinical examination was performed, and necessary investigations were carried out. Relevant investigations like complete blood counts, urine examination, serum electrolytes. The results were analysed to assess the aetiology, risk factors, and the pattern of clinical and radiological profile.

\section{Statistical Analysis}

Data was recorded in the form of tables and graphs. The data was analyzed using SPSS program version 21 software. The data was summarized and tabulated accordingly to analyze frequencies.

\section{RESULTS}

In the present study, we observed that majority of the cases presented with accelerated hypertension were males $(56.25 \%)$, followed by $43.75 \%$ female cases. We observed that majority of the cases of accelerated hypertension belonged to $56-65$ years of age group (29.16\%), followed by 46-55 years $(26.16 \%)$, and $66-75$ years $(16.66 \%)$. The mean age of the study subjects was $57.41 \pm 14.12$ years. We assessed their hypertensive status and diabetic status, we observed that majority of the cases were known cases of hypertension (67.70\%), and $12.5 \%$ cases were known cases of diabetes mellitus and $4.16 \%$ cases were known cases of ischaemic heart disease.

\begin{tabular}{|c|c|c|}
\hline Blood Pressure & Mean & Standard Deviation \\
\hline Mean SBP & 183 & 24.7 \\
\hline Mean DBP & 99.68 & 18.03 \\
\hline \multicolumn{2}{|c|}{ Table 1. Distribution of Study Subjects According to their Blood } \\
Pressure
\end{tabular}

\begin{tabular}{|c|c|c|}
\hline Clinical Presentation & Number of Cases & Percentage \\
\hline Headache & 78 & $81.25 \%$ \\
\hline Giddiness & 36 & $37.5 \%$ \\
\hline Chest pain & 34 & $35.42 \%$ \\
\hline Dyspnoea & 19 & $19.79 \%$ \\
\hline Limb weakness & 15 & $15.62 \%$ \\
\hline Oedema & 10 & $10.41 \%$ \\
\hline Pallor & 11 & $11.45 \%$ \\
\hline \multicolumn{3}{|c|}{ Table 2. Distribution of Study Subjects According to their Clinical } \\
Presentation \\
\hline
\end{tabular}

\begin{tabular}{|c|c|c|}
\hline ECG Changes & Number of cases & Percentage \\
\hline QT prolongation & 22 & $22.91 \%$ \\
\hline T wave inversion & 18 & $18.75 \%$ \\
\hline ST segment depression & 15 & $15.62 \%$ \\
\hline U waves & 7 & $7.29 \%$ \\
\hline Tachycardia & 21 & $21.87 \%$ \\
\hline Bradycardia & 3 & $3.1 \%$ \\
\hline Other arrythmias & 2 & $2.08 \%$ \\
\hline \multicolumn{2}{|c|}{ Table 3. Distribution of Study Subjects According to their } \\
Electrocardiographic Changes \\
\hline
\end{tabular}

In the present study, we observed that majority of the study subjects presented with headache as a presenting complaint (81.25\%), followed by giddiness among 37.5\% cases, $35.42 \%$ cases presented with chest pain, $19.79 \%$ cases presented with dyspnoea, weakness in limbs among $15.62 \%$ cases and oedema among $10.41 \%$ cases. We assessed the systolic and diastolic blood pressures of all the study subjects at presentation. We observed that the mean systolic blood pressure on admission was $183 \pm 24.7 \mathrm{mmHg}$, while mean diastolic blood pressure on admission was 99.68 \pm 18.03 mmHg. Serum levels of TC, TG, HDL and LDL were $182 \pm 3.4$, $143.62 \pm 6.3,49.68$ and $95.83 \pm 7.8 \mathrm{mg} / \mathrm{dL}$, respectively, in hypertensive subjects.

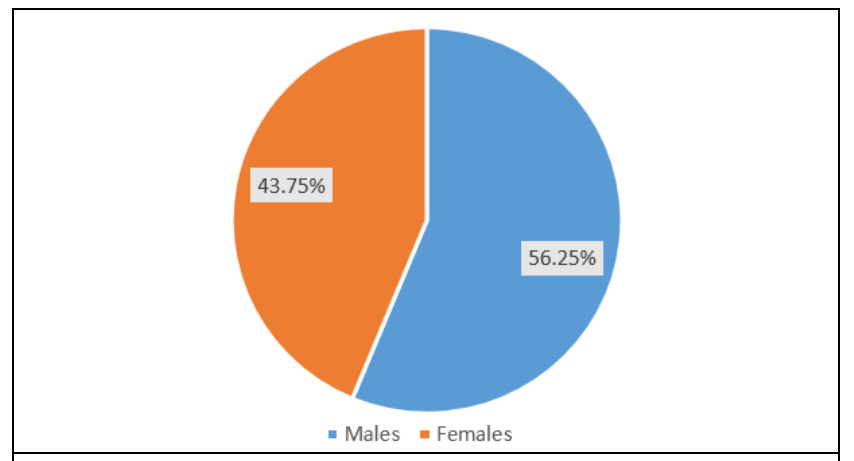

Figure 1. Distribution of Study Subjects According to their Gender
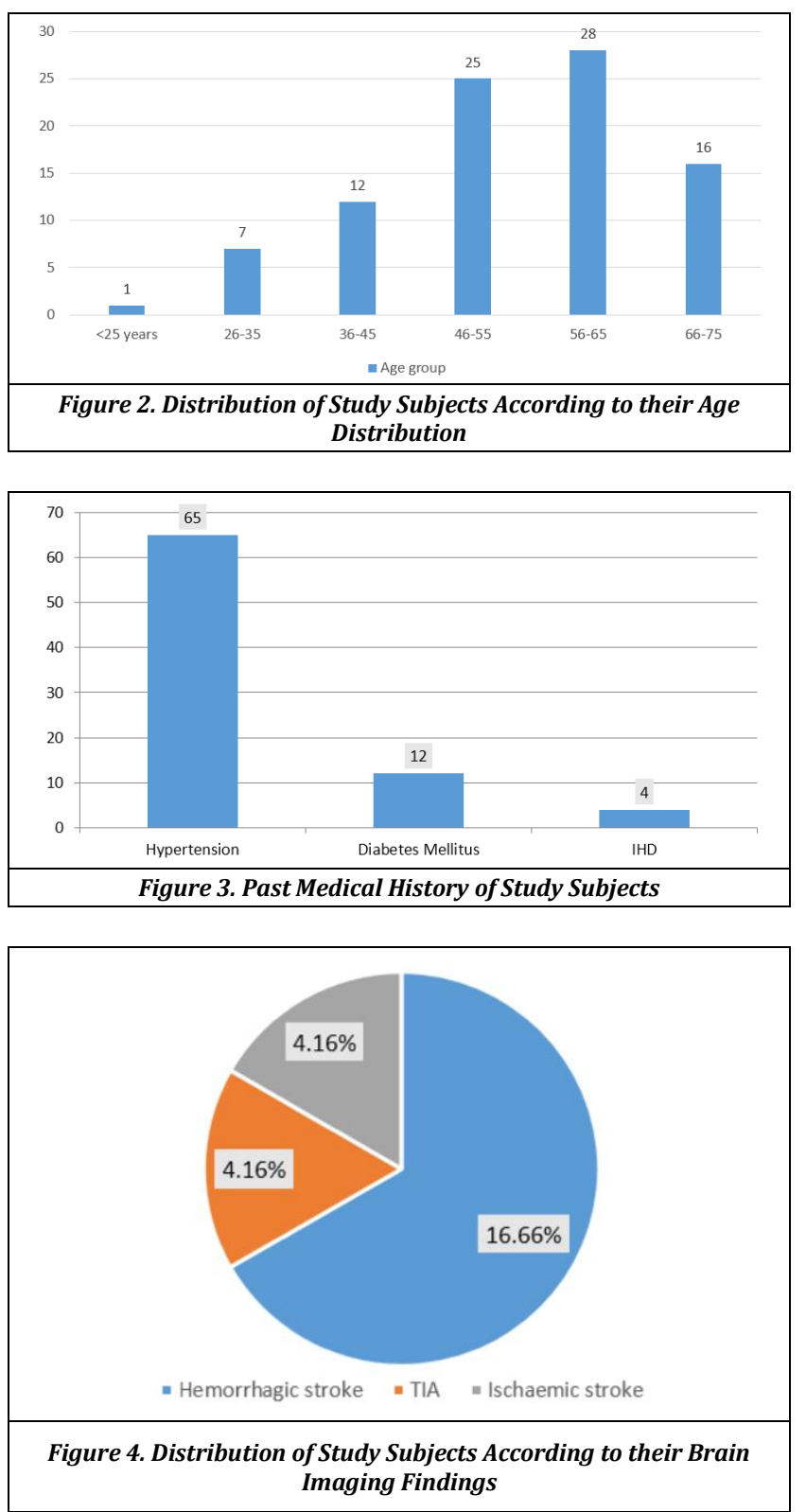

The most common ECG abnormality associated with stroke was prolonged QTc interval (22.91\%) followed by T wave inversion $(18.75 \%)$, ST segment changes (15.62\%), 
sinus tachycardia (21.87\%), U-waves (7.29\%), bradycardia (3.1\%), and other arrythmias (2.08\%).

\section{DISCUSSION}

In the present study, we observed that majority of the cases presented with accelerated hypertension were males (56.25\%), followed by $43.75 \%$ female cases. The current cross-sectional analytical study was conducted at tertiary health care centreto assess cases presenting to outpatient department and emergency department with hypertensive emergencies. Majority of the study subjects reported in the present study were males (66\%) as compared to proportion of female cases. Martin et al in their study among cases presented with hypertensive crises, reported that $55 \%$ of total patients were males, while Zampaglione et al. ${ }^{20}$ in their study also reported similar kind of finding, they reported that majority of the study subjects were males. In the current study, we observed that majority of the cases of accelerated hypertension belonged to 56-65 years of age group (29.16\%), followed by 46-55 years (26.16\%), and 66-75 years $(16.66 \%)$. The mean age of the study subjects was $57.41 \pm 14.12$ years.

We assessed their hypertensive status and diabetic status, we observed that majority of the cases were known cases of hypertension $(67.70 \%)$, and $12.5 \%$ cases were known cases of diabetes mellitus and $4.16 \%$ cases were known cases of ischaemic heart disease. Garcia GM in their study observed that $65.9 \%$ subjects were known cases of hypertension. While Zampanglione et al, ${ }^{21}$ observed $92 \%$ of previously diagnosed hypertensive cases. Martin et al. ${ }^{20}$ in their study observed the prevalence of diabetes mellitus among their cases as $26 \%$. Martin et al, 20 in their study studied the clinical presentation of the study subjects. They reported that the commonest presenting symptoms were neurological deficits (48\%), dyspnoea (25\%) and chest pain $(18 \%)$ as the most common clinical presentation. While Zampaglione et al,21 observed chest pain among $27 \%$ cases, followed by dyspnoea among $22 \%$ cases and neurological deficits was observed among $21 \%$. Neurological deficits in their study varied from

hemiparesis among $75 \%$ of the cases with neurological deficits, convulsion among $16.6 \%$ cases, and visual deficits among $8.3 \%$ cases. Hemiparesis accounted for the largest group of patients with neurological deficit

We assessed the systolic and diastolic blood pressures of all the study subjects at presentation. We observed that the mean systolic blood pressure on admission was $183 \pm 24.7$ mmHg, while mean diastolic blood pressure on admission was $99.68 \pm 18.03 \mathrm{mmHg}$. Martin et al. ${ }^{23}$ In their study reports a mean systolic blood pressure of $193 \pm 26 \mathrm{mmHg}$ in their patients and a mean diastolic blood pressure of $129 \pm 12$ mmHg. The most common ECG abnormality associated with stroke was prolonged QTc interval $(22.91 \%)$ followed by $\mathrm{T}$ wave inversion (18.75\%), ST segment changes (15.62\%), sinus tachycardia (21.87\%), U-waves $(7.29 \%)$, bradycardia (3.1\%), and other arrythmias (2.08\%). In the study conducted by Srikanth Tandur et al, ${ }^{21}$ observed QTc prolongation in their study as the most common finding (31.08\%, i.e., 23 of 74 cases) which was followed by $\mathrm{T}$ wave inversion ( $24.32 \%$ i.e., 18 cases), and ST segment changes were observed among ( $20.27 \%$, i.e., 15 cases).

\section{CONCLUSIONS}

From the present study, we observed that, cases presenting with cerebrovascular disorders (CVA) often have abnormal electrocardiography pattern with or without presence of any obvious known organic heart disease or electrolyte imbalance. The most common ECG changes observed in the current study were prolonged QTc interval, T wave inversion, ST segment depression and tachycardia.

\section{ACKNOWLEDGEMENT}

Authors acknowledge Dr. D. M. Patel and Dr. Nitin Jadhav for revising the linguistic content of this manuscript. The authors thank all the study participants.

\section{REFERENCES}

[1] Reddy KS. Cardiovascular disease in non-Western countries. N Engl J Med 2004;350(24):2438-40.

[2] Murray CJ, Lopez AD. Global mortality, disability and the contribution of risk factors: Global Burden of Disease Study. Lancet 1997;349(9063):1436-42.

[3] Keith NM, Wagener HP, Barker NW. Some different types of essential hypertension: their course and prognosis. Am J Med Sci 1939;197:332-43.

[4] Laragh J. Laragh's lessons in pathophysiology and clinical pearls for treating hypertension. Am J Hypertens 2001;14(9 Pt 1):837-54.

[5] McRae RP Jr, Liebson PR. Hypertensive crisis. Med Clin North Am 1986;70(4):749-67.

[6] Vidt DG. Current concepts in treatment of hypertensive emergencies. Am Heart J 1986;111(1):220-5.

[7] Vital and health statistics: detailed diagnoses and procedures for patients discharged from short-stay hospitals: United States, 1983-1990. Hyattsville, MD: National Center for Health Statistics, 1997.

[8] Bennett NM, Shea S. Hypertensive emergency: case criteria, sociodemographic profile and previous care of 100 cases. Am J Public Health 1988;78(6):636-40.

[9] Tisdale JE, Huang MB, Borzak S, et al. Risk factors for hypertensive crisis: importance of out-patient blood pressure control. Fam Pract 2004;21(4):420-4.

[10] Tumlin JA, Dunbar LM, Oparil S, et al. Fenoldopam, a dopamine agonist, for hypertensive emergency: a multicenter randomized trial: fenoldopam Study Group. Acad Emerg Med 2000;7(6):653-62.

[11] Shea S, Misra D, Ehrlich MH, et al. Predisposing factors for severe, uncontrolled hypertension in an inner-city minority population. N Engl J Med 1992;327(11):77681. 
[12] Calhoun DA, Oparil S. Treatment of hypertensive crisis. N Engl J Med 1990;323(17):1177-83.

[13] Joshi P, Islam S, Pais P, et al. Risk factors for early myocardial infarction in South Asians compared with individuals in other countries. JAMA 2007;297(3):28694.

[14] Mora S, Glynn RJ, Ridker PM. High-density lipoprotein cholesterol, size, particle number, and residual vascular risk after potent statin therapy. Circulation 2013;128(11):1189-97.

[15] Liu Y, Zhang B, Chen JY, et al. The relationship between fasting triglyceride level and prevalence and severity of angiographic coronary artery disease in 16,650 patients from the TRUST study in the statins era. Eur Heart J 2013;34(Suppl 1):P1550.

[16] Moniruzzamani AT, Rahmani S, Acharyyai A, et al. Prevalence of hypertension among the Bangladeshi adult population: a meta-analysis. Regional Health Forum. 1. Vol. 17. Geneva: World Health Organization, 2013.
[17] Akhtaruzzaman M, Khan MNI, Islam SN. Nutrition, Health and Demographic Survey of Bangladesh-2011. Dhaka, Bangladesh: Institute of Nutrition and Food Science University of Dhaka: 2013. [Accessed May 12, 2014].

[18] World Health Organization. Non-Communicable Disease Risk Factor Survey Bangladesh 2010. Ministry of Health and Family Welfare, Bangladesh: 2010. [Accessed May 12, 2014].

[19] Saquib N, Saquib J, Ahmed T, et al. Cardiovascular diseases and type 2 diabetes in Bangladesh: a systematic review and meta-analysis of studies between 1995 and 2010. BMC Public Health 2012;12:434

[20] Zampaglione B, Pascale C, Marchisio M, et al. Hypertensive urgencies and emergencies. Prevalence and clinical presentation. Hypertension 1996;27(1):144-7.

[21] Tandur S, Sundaragiri S. A study of electrocardiographic changes in acute cerebrovascular accidents. Int J Med Sci Public Health 2016;5(12):2560-5. 